\title{
Double grating formation in anisotropic photorefractive crystals
}

\author{
Hsin-Yu Li and Demetri Psaltis \\ Department of Electrical Engineering, California Institute of Technology, Pasadena, California 91125
}

(Received 6 September 1991; accepted for publication 21 October 1991)

Double gratings form in anisotropic photorefractive crystals when two plane waves intersecting inside the crystal have both extraordinary and ordinary wave components. The effect can be observed by reading out the two gratings with extraordinary or ordinary light. Experiments confirm the theoretical predictions. The relevance of these results in using photorefractive crystals for holographic storage is discussed.

\section{INTRODUCTION}

Holographic gratings are recorded in photorefractive crystals such as $\mathrm{LiNbO}_{3}$ when exposed to coherent interference patterns. ${ }^{1}$ In the simplest case, two incident plane waves intersect inside the crystal and form a single grating. The change of index in photorefractive crystals such as $\mathrm{LiNbO}_{3}$ depends on the first-order electro-optic (LO) effect. Many crystals that exhibit this property (e.g., $\mathrm{LiNbO}_{3}$ ) are anisotropic. Hence plane waves that are not eigenmodes split into two components, the ordinary wave ( $o$ wave) and the extraordinary wave ( $e$ wave). When this occurs, double gratings are written for each plane wave pair, each grating being associated with one of the eigenmodes (polarizations).

In using photorefractive crystals for optical storage, a commonly used technique is angle multiplexing where the reference beam angle is changed for each recorded hologram (frame). Five hundred holograms have been stored in the same location using this method. ${ }^{2}$ Cross-talk between the various holograms is avoided by the angular (Bragg) selectivity of thick holograms. The width of the Bragg matching angle (which depends on the crystal thickness) dictates how far apart the reference beam angle needs to change from frame-to-frame to avoid cross-talk between the holograms.

In the usual setup for recording holograms in $\mathrm{LiNbO}_{3}$ crystals, the $c$ axis lics on the plane of incidence (defined by the wave vectors of the signal and reference beams), and the optical (electric) field is polarized either in-plane (horizontal polarization) or perpendicular (perpendicular polarization) to the plane of incidence. When the signal and reference beams are plane waves, they are eigenmodes, and the beams do not split upon refraction. Therefore, a single grating is written, and the problem does not arise in this simple case. Cross-talk then depends only on the width of the Bragg matching angles. In most other cases, however, the potential for double grating formation exists.

An example of particular interest is recording spatially multiplexed hologram. The amount of information that can be stored at a single location is approximately $10^{9}$ bits, limited by the size of the images stored $\left[\approx 10^{3} \times 10^{3}\right.$ pixels on a single spatial light modulator (SLM)] and the number of images that can be superimposed $\left(\approx 10^{3}\right.$ images for the dynamic range of photorefractive holograms). In order to expand the storage capacity, it is advantageous to divide the crystal into several smaller regions, and then store angularly multiplexed images at each location. In this case, the incident plane (the plane formed by the reference and signal beams) changes orientation with respect to the crystal as the writing beams are scanned from one location to another. In general, the polarization of the reference beam and/or the signal beam will be in directions that are not eigenmodes, which gives rise to double gratings. Upon reconstruction of the holograms by scanning the reference beam angle, it is possible for each of the gratings to be Bragg matched at more than one angle. Thus there are three angles at which each hologram (consisting now of a double grating pair) can be potentially Bragg matched, and this results in additional cross-talk between the various holograms when we try to do angle multiplexing.

In this paper we show how such cross-talk may occur if the light polarization is not selected correctly. Comparisons between theory and experiment are given, as well as a discussion of possible applications.

\section{THEORY}

The geometry of our experiment is shown in Fig. 1. The coordinates $x, y$, and $z$ are the crystal axes. The $c$ axis $(z)$ is tilted at an angle $\varphi$ with respect to the plane of incidence. The direction of propagation of the signal beam is in the $x$ direction, perpendicular to the surface of the crystal, the $y-z$ plane. The reference beam is at an angle $\theta$ with respect to the signal beam. The recording beams are horizontally polarized, and have both $e$-wave and $o$-wave components. The angle between $\mathbf{k}_{1}$ and $\mathbf{k}_{2}$ is denoted as $\theta$. This geometry was chosen only for convenience of analysis. The results are similar for other recording geometries.

Upon entering the crystal, the two eigenmodes refract according to Snell's law. The direction of the refracted beam depends on the index of the crystal, which can be found from the normal surfaces of the $e$ and $o$ waves. These are

$$
\frac{x^{2}}{n_{o}^{2}}+\frac{y^{2}}{n_{o}^{2}}+\frac{z^{2}}{n_{o}^{2}}=1
$$

for the ordinary wave normal surface, and

$$
\frac{x^{2}}{n_{e}^{2}}+\frac{y^{2}}{n_{e}^{2}}+\frac{z^{2}}{n_{o}^{2}}=1
$$

for the cxtraordinary wave normal surface. 


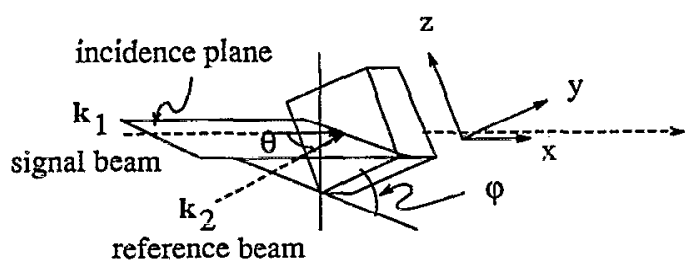

(a)

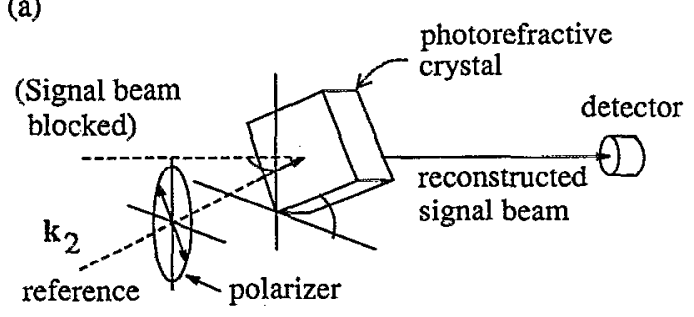

(b)

FIG. 1. Geometry of recording and measurement. (a) Recording geometry. (b) Setup for experiment in Figs. 6 and 7.

The refracted beams remain within the incident plane since the signal beam is perpendicular to the crystal surface. Therefore we need only consider the intersections of the two normal surfaces with the incident plane. The intersections are two ellipses, as illustrated in Fig. 2. They do not touch each other because the incident plane is at an angle $\varphi$ with respect to the crystal axis $z$. The equations for the two ellipses are readily found to be:

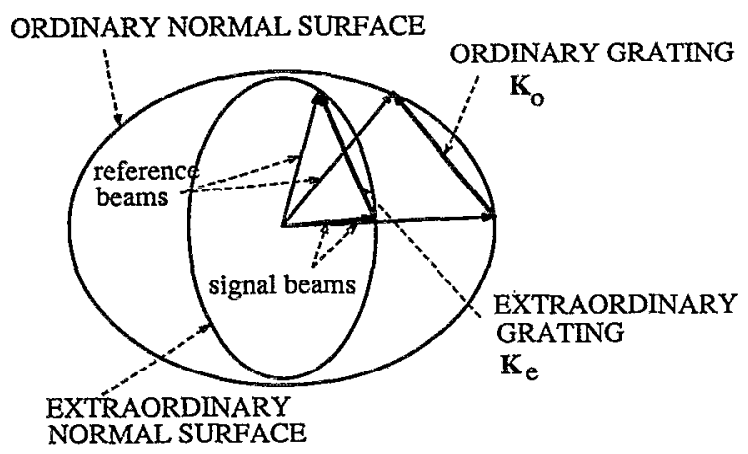

(a) Recording of gratings.

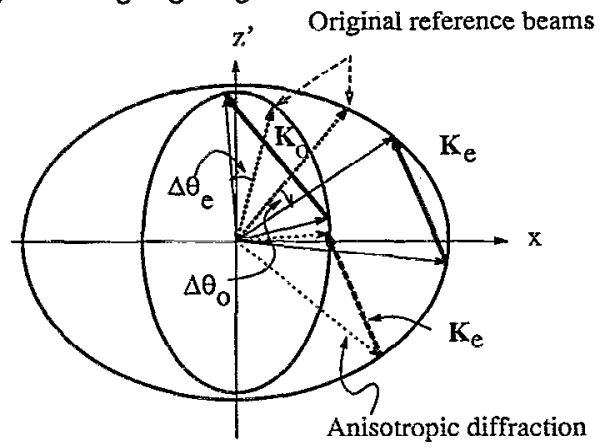

(b) reconstruction of signal beams.

FIG. 2. Normal surfaces and grating vectors.

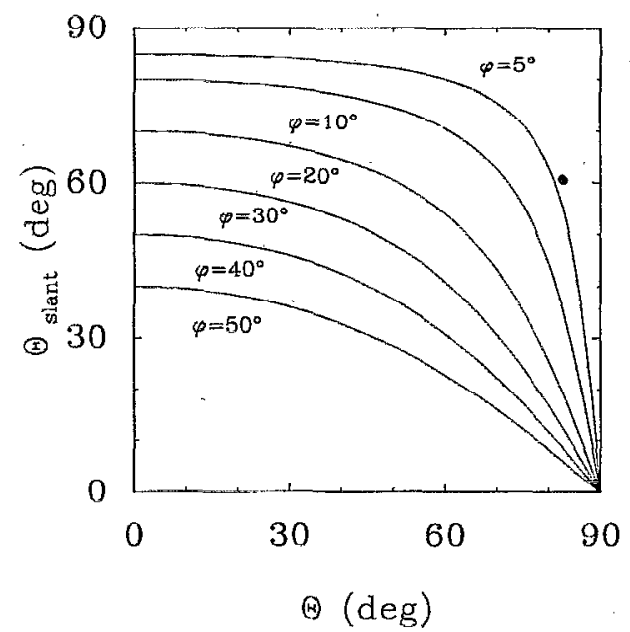

FIG. 3. Slant angle between $e$-wave polarization and direction normal to incident plane.

$$
\frac{x^{2}}{n_{o}^{2}}+\frac{z^{2}}{n_{o}^{2}}=1
$$

for $o$ waves, and

$$
\frac{x^{2}}{n_{e}^{2}}+\left(\frac{\sin ^{2} \varphi}{n_{e}^{2}}+\frac{\cos ^{2} \varphi}{n_{o}^{2}}\right) z^{\prime 2}=1
$$

for $e$ waves. ( $x$ lies in the incident plane. $z^{\prime}$ is the direction on the incident plane perpendicular to $x$.)

Each beam splits into two eigenmodes according to Snell's law. The situation is shown in Fig. 2. For our particular case, the two components for the signal beam are actually in the same direction since we have selected for convenience the direction of the signal beam to be along the crystal axis $x$. (In Fig. 2, they are separated for clarity.) The two eigenmodes are perpendicular to each other in polarization, but it should be noted that the eigenmode polarizations are in general neither parallel nor perpendicular to the crystal axes. One of the reasons for choosing the signal beams to propagate along the direction of a crystal axis $x$ is because the eigenmode polarization directions are exactly along the other two crystal axes, $y$ and $z$. Figure 3 shows the calculated slant angle, defined as the angle between the $e$-wave polarization and the direction normal to the plane of incidence, for several values of $\varphi$. The derivation of the slant angle is given in Appendix A.

The four refracted signal and reference beams inside the crystal form interference patterns which write gratings on the crystal via the photorefractive effect.The $e$-wave components form the grating $\mathbf{K}_{e}$, which we will call the extraordinary grating. The $o$-wave components form the, grating $\mathbf{K}_{o}$, which we will call the ordinary grating. From the geometry of Fig. 2, and the application of Snell's law, we can calculate the directions of the refracted beams, and hence the two gratings:

$$
\mathbf{K}_{e}=\left(n_{e} \sqrt{1-\sin ^{2} \theta\left(\frac{\sin ^{2} \varphi}{n_{e}^{2}}+\frac{\cos ^{2} \varphi}{n_{o}^{2}}\right)}-n_{e}, \sin \theta\right)
$$




$$
\mathbf{K}_{o}=\left(n_{o} \sqrt{1-\frac{\sin ^{2} \theta}{n_{o}^{2}}}-n_{o}, \sin \theta\right) .
$$

In addition to the two gratings $\mathbf{K}_{e}$ and $\mathbf{K}_{o}$, we also get gratings from interference between the $e$-wave/o-wave component of the reference beam and the $o$-wave/e-wave component of the signal beam. However these two "cross gratings" are much weaker than $\mathbf{K}_{o}$ and $\mathbf{K}_{e}$ since the $o$ wave and $e$-wave modes are polarized orthogonally to each other for the same beam, and are almost perpendicular to each other for the signal and reference beams. Estimates of the modulation depths for $\mathbf{K}_{e}, \mathbf{K}_{v}$, and the cross gratings show that the modulation depths of the cross grating is less than $5 \%$ of that of $\mathbf{K}_{c}$ and $\mathbf{K}_{o}$. (In fact, it turns out that the $e$-wave of the signal beam and the $o$-wave of the reference beam are exactly perpendicular to each other. See Appen$\operatorname{dix}$ A.)

Once the gratings are recorded in the crystal, the signal beam can be reconstructed by the reference beam. The angle at which the signal can be reproduced is determined by the Bragg condition $\mathbf{k}_{1}=\mathbf{k}_{2}+\mathbf{K}{ }^{3}$ Since we have two gratings, it is possible to satisfy the Bragg condition at more than one angle. In addition to the original recording angle (where $\mathbf{K}_{e}$ is read out by the $e$ wave and $\mathbf{K}_{o}$ is read out by the $o$ wave), we can get Bragg matching when $\mathbf{K}_{e}$ is read out by the $o$ wave and also when $K_{o}$ is read out by the $e$ wave, as shown in Fig. 2(b). It is also possible that one of the two gratings can also satisfy the Bragg condition for anisotropic diffraction. ${ }^{4-7}$ The anisotropic Bragg matching condition for $\mathbf{K}_{e}$ is also shown in Fig. 2(b). We will not consider anisotropic diffraction for the rest of this paper, however, it can be treated in a way completely analogous to the isotropic case we will treat.

The angles where the additional Bragg matching conditions occur are very close to the original recording angle [the dotted lines in Fig. 2(b)]. The deviation of the Bragg angle of the reconstructing beam from the original recording angle, denoted as $\Delta \theta_{e}$ (for $e$ wave) and $\Delta \theta_{o}$ (for $o$ wave), can be calculated as a function of $\theta$ and $\varphi$ from the geometry of Fig. 2. The calculation is shown in Appendix $\mathrm{B}$, and the result is plotted in Fig. 4 for $\mathrm{LiNbO}_{3}$, where $n_{e}=2.208$ and $n_{o}=2.286$. Note that $\Delta \theta$ depends only on the orientation of the crystal with respect to the writing beams (i.e., $\theta$ and $\phi$ ). Inside the crystal, the Bragg angle is

$$
\Delta \theta_{\text {Bragg, inside }} \approx \Lambda / L=\frac{\lambda}{2 n L \sin \left(\theta_{\text {in }} / 2\right)},
$$

where, $\theta_{\text {in }}$ here is the angle inside the crystal.Outside the crystal, because of Snell's law, we have

$$
\Delta \theta_{\mathrm{Bragg}} \approx \frac{\sqrt{n^{2}-\sin ^{2} \theta}}{\cos \theta} \Delta \theta_{\mathrm{Bragg}, \text { inside }},
$$

where $n$ is the index of refraction, and $L$ is the interaction length. For $\mathrm{LiNbO}_{3}(n \approx 2.2)$ with $L=8 \mathrm{~mm}$, and using $\lambda=488 \mathrm{~nm}$ and $\theta=30^{\circ}$, we get $\Delta \theta_{\text {Bragg }}$ about $0.01^{\circ}$. For $\varphi=30^{\circ}$ and $\theta=30^{\circ}$, we get from Fig. $4 \Delta \theta_{e} \approx \Delta \theta_{o} \approx 0.25^{\circ}$, which is much larger than $\Delta \theta_{\mathrm{Bragg}}$. This implies that in a system where we store images using angular multiplexing,

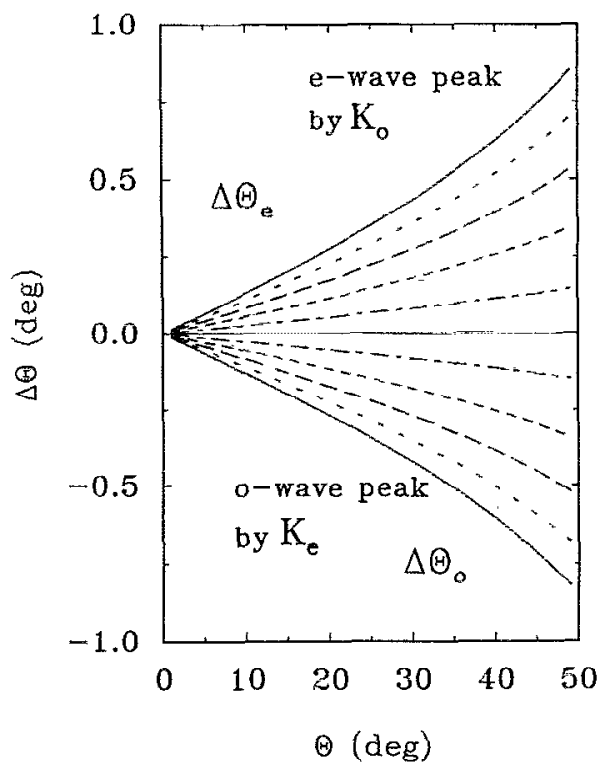

FIG. 4. Deviation angle of the side-peaks from the recording reference beam angle as a function of $\theta$ for various values of $\varphi$.

the double grating will interfere with an image stored approximatcly 25 positions away.

\section{EXPERIMENTS}

The experimental setup is shown in Fig. 1(b), with $\theta=34^{\circ}$ and $\varphi=30^{\circ}$. Both signal and reference beams are polarized in the plane of incidence. We record a hologram in a $\mathrm{LiNbO}_{3}$ crystal (grown by Deltronics; $20 \times 20 \times 8 \mathrm{~mm}$, $0.01 \% \mathrm{Fe}$ doping) using two plane waves, then block the signal beam. The detected intensity for the reconstructed beam (which has both $e$-wave and $o$-wave components) as a function of $\Delta \theta$ is shown in Fig. 5. As expected, there are three peaks. In addition to one at the original angle ( $\Delta \theta$ $=0^{\circ}$ ), there are two side-peaks. The side-peaks are measured to be at $\Delta \theta_{\mathrm{e}}=+0.36^{\circ} \pm 0.01^{\circ}$ and $\Delta \theta_{o}=$ $-0.35^{\circ} \pm 0.01^{\circ}$. The theoretical prediction is $\pm 0.31^{\circ}$ for both $\Delta \theta_{e}$ and $\Delta \theta_{o}$. The central peak in Fig. 5 has both $e$-wave and $o$-wave components, while the side-peak on the right is an $e$ wave, and the peak on the left is an $o$ wave.

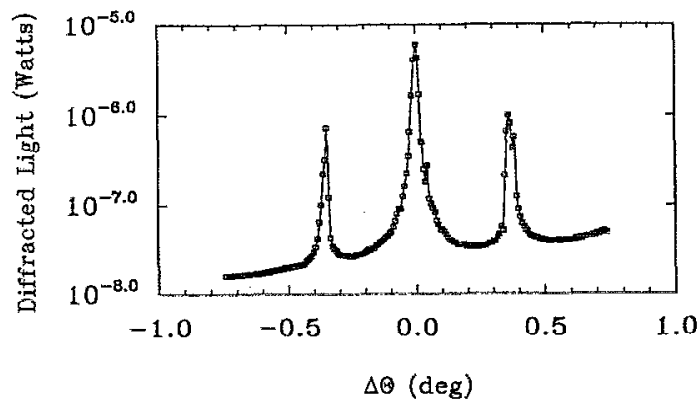

FIG. 5. Measured intensity of reconstructed beam as a function of deviation of reference angle. Reference beam has both $e$-wave and $o$-wave components. 


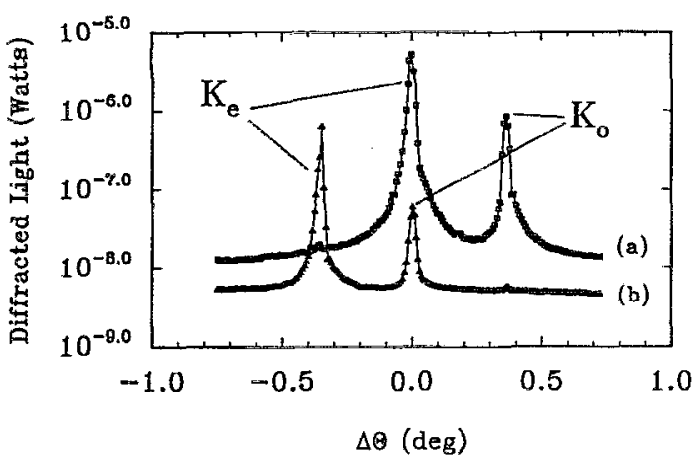

FIG. 6. Measured intensity of reconstructed beam as a function of deviation of reference angle. (a) Reference beam has e wave only. (b) Reference beam has $O$ wave only.

If we reconstruct the signal beam with a reference beam that has only $e$-wave components, we Bragg match $\mathbf{K}_{e}$ at one angle (the original recording angle), and $\mathbf{K}_{o}$ at a different angle. This gives us two peaks (both of which are $e$ waves), as shown in Fig. 6(a). Similarly, we get two peaks when the reference beam has only $o$-wave components [Fig. 6(b)].

The relative heights of the two peaks shown in Figs. 6(a) and (b) can be estimated as follows. According to Kogelnik's theory of volume holograms, ${ }^{3}$ the diffraction efficiency of a thick phase grating is proportional to the square of the index change $\Delta n$ of the grating. This in turn is proportional to the modulation depth of the interference pattern for weak holograms. We can measure experimentally the $e$-wave components of the signal $\left(I_{e 1}\right)$ and reference beam $\left(I_{e 2}\right)$, and the $o$-wave components of the signal $\left(I_{o 1}\right)$ and reference beam $\left(I_{o 2}\right)$ that are transmitted through the crystal. From this we estimate the ratio of the modulation depths of $\mathbf{K}_{e}$ and $\mathbf{K}_{o}$ to be $\sqrt{I_{e 1} I_{e 2} / I_{o 1} I_{o 2}}$ $=2.94$, so the height of the two peaks [for both Figs. 6(a) and $6(\mathrm{~b})]$ should have a ratio of $2.94^{2}=8.67$. (Strictly speaking, the diffracting efficiency also depends on twowave-mixing coupling coefficients ${ }^{8}$ that are functions of $\theta$ and beam polarization. However $\Delta \theta$ is $<0.5^{\circ}$, so the change is negligible.) The actual measured ratios were 5.96 for the two $e$-wave peaks [Fig. 6(a)] and 10.37 for the two 0 -wave peaks [Fig. $6(\mathrm{~b})]$.

Instead of using plane waves, we can also record images. We recorded two images on a photorefractive crystal using the setup shown in Fig. 7. The images 1 and 2 are photographic plate transparencies. The incident plane wave is split by the polarizing beamsplitter (PB) into horizontal and vertical components. Each illuminates one of the transparencies, and the two images are recombined by a second $\mathrm{PB}$. The hologram is recorded with a reference beam that has both horizontal (e-wave) and vertical ( $o$ wave) polarization. The first image consisted only of $e$ wave, and the second image consisted only of $o$ wave. After the hologram was recorded, we observed the image reconstructed by the same reference beam used for recording. As we change the polarization of the reference beam, images 1 and 2 appear and disappear in turn, since the former is

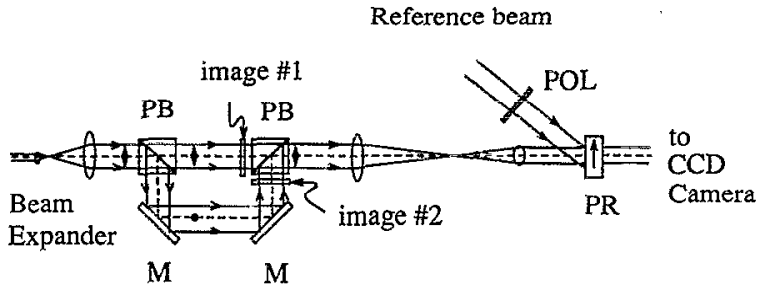

PB: Polarizing Beamsplitter POL: Polarizer PR: Photorefractive Crystal M: Mirror

FIG. 7. Setup for recording two images of orthogonal polarizations. Image 1 has only $o$-wave component. Image 2 has only e-wave component.

recorded as $\mathbf{K}_{e}$ gratings, and the latter is recorded as $\mathbf{K}_{0}$ gratings. The results are shown in Fig. 8. Figure 8(a) shows the reconstructed image when both $o$ - and $e$-wave components are present in the reference beam. Figures 8 (b) and 8(c) show that only one of the two images appears when the reference beam is $o$ wave or $e$ wave only. Note that there is some cross-talk: There is a trace of image 2 in image 1 . This is probably because the polarizations were not exactly aligned with the $c$ axis of the crystal, so there was some $o$-wave component in the $e$-wave image of 1. The pictures were captured by a CCD camera, then taken off the monitor by a polaroid camera.

\section{DISCUSSION AND CONCLUSION}

Double gratings are written in photorefractive crystals when the recording beams have both $e$-wave and $o$-wave components. As a result, it is possible to satisfy the Bragg matching condition at more than one reference beam angle. This can be a problem when we record multiple holograms by angle multiplexing. When we read out one of the holograms recorded at a new reference beam angle, the new reference beam can coincide with one of the side-peaks of previous holograms, even though the new reference beam angle is separated from the other reference beam angles by more than several $\Delta \theta_{\mathrm{Bragg}}$.

To avoid this problem, we might limit the range of $\theta$ and $\phi$ that we are working with, so that $\Delta \theta_{e}$ and $\Delta \theta_{o}$ are larger than the range of reference beam angles that we want to use, so that the side-peaks and central-peaks effectively coincide.

As an example, consider a crystal that has thickness of $8 \mathrm{~mm}$, index $n=2.2$, and $\theta=30^{\circ}$. We have $\Delta \theta_{\mathrm{Bragg}} \approx 0.01^{\circ}$ from Eq. (8). Taking twice this value as the separation between reference beam angles, storing 1000 holograms would require a range of $\theta$ of about $20^{\circ}$. We can calculate $\Delta \theta_{e}$ and $\Delta \theta_{o}$ for various values of $\varphi$ at $\theta=20^{\circ}, \theta=30^{\circ}$, and $\theta=40^{\circ}$ (see Appendix B). The result is plotted in Fig. 9. In $\mathrm{LiNbO}_{3}$ we would like to work near $\varphi=0$. where the $c$ axis is close to the incident plane, and the diffraction efficiencies are high. $\Delta \theta_{e}$ and $\Delta \theta_{o}$ in this region are about $1^{\circ}$, which means that we can place in about $0.5^{\circ} / 0.02^{\circ}=25$ holograms before we start hitting the side-peaks. In this 


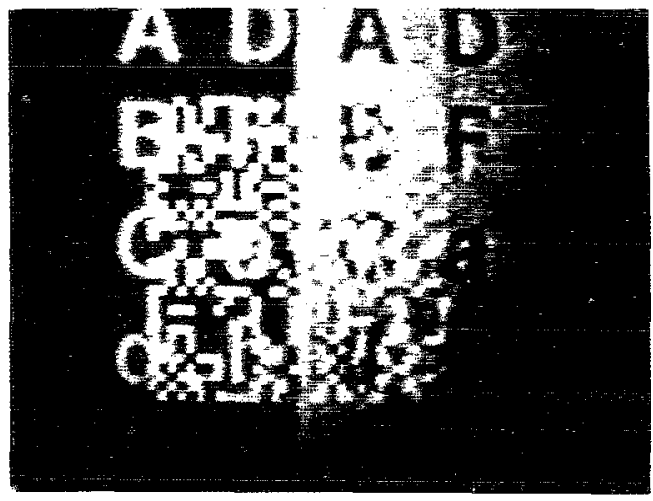

(a)

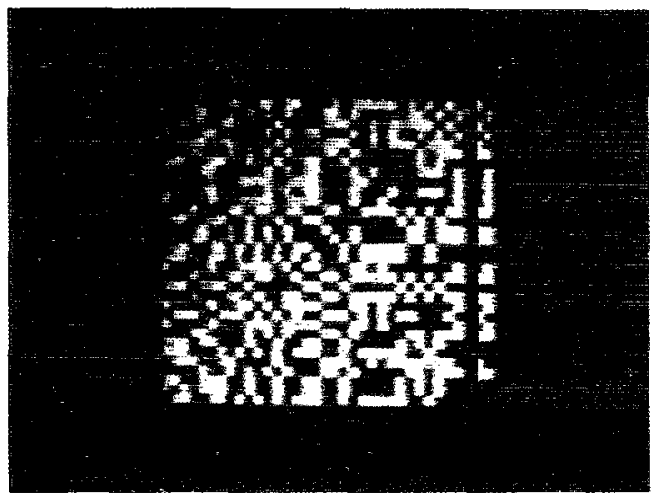

(b)

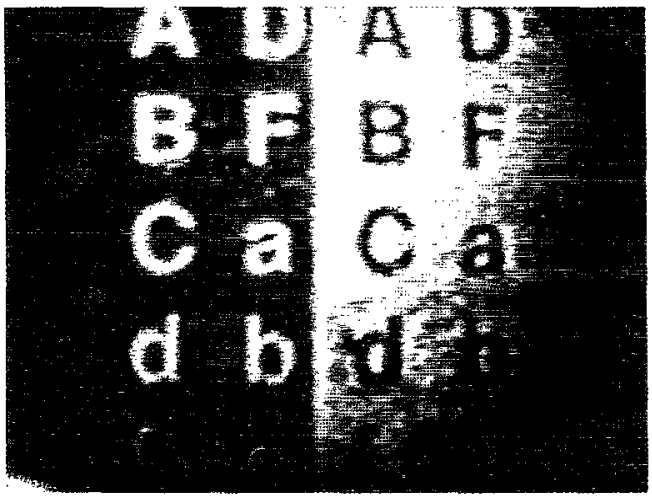

(c)

FIG. 8. (a) Reconstructed image when both $e$ and $o$ waves are present in reference beam. (b) Reference beam has $e$ wave only. (c) Reference beam has $o$ wave only. The images were captured by a CCD camera, then taken off the monitor by a polaroid camera.

example, we would have to record 25 adjacent, angularly multiplexed holograms, and then leave a blank of 25 slots for the cross-gratings. It is in principle possible to use the system in Fig. 7, to record two separate images in two polarizations in such a way that the cross-gratings that appear in the "empty" slots would actually be new images. With this scheme, there is no loss in storage density, at the expense of considerable added complexity. As $\varphi$ increases, the number of adjacent holograms that can be recorded before we must leave a gap decreases.

The second alternative is to squeeze the side-peaks as close as possible around the central-peak. As shown in Fig.
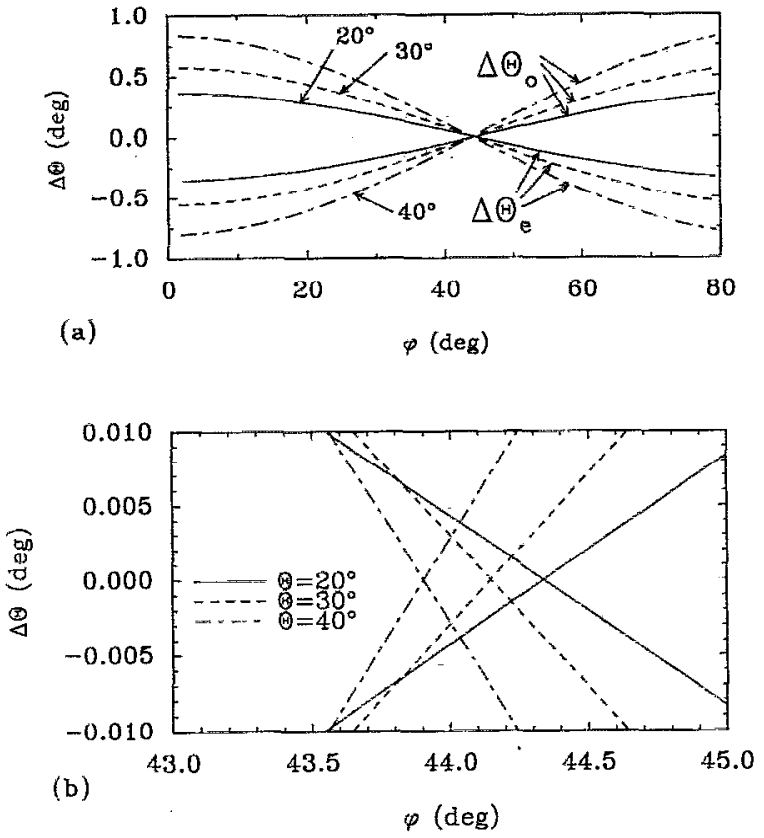

FIG. 9. (a) Deviation angle of the side-peaks as a function of $\varphi$ for $\theta=20^{\circ}, 30^{\circ}$, and $40^{\circ}$. (b) Enlargement of the region where $\Delta \theta_{e}$ and $\Delta \theta_{o}$ are zero.

9 (b), around $\varphi \approx 44.1^{\circ}$, all three peaks actually coincide. However, for the separation of the side-peaks and centralpeaks to be much smaller than $\Delta \theta_{\text {Bragg }}$ throughout the whole working range of $\theta$ (about $20^{\circ}$ to $40^{\circ}$ ), we see that we are limited to a range of $\varphi$ (tilt) of only one or two degrees. This puts a rather strict constraint on system design. In general, either we have to limit the number of holograms we can store, or limit the amount of tilting allowed. The example above shows that neither case is very satisfactory. There is, however, one situation where the side-peak problem does not occur for the range of angles used in angle multiplexing. This is when we go to reflection type holograms, where $\theta>90^{\circ}$. In this case, calculations similar to those shown in Figs. 4 and 9 show that the $o$-wave peak disappears $\left(\Delta \theta_{o} \rightarrow \infty\right)$ and the $e$-wave peak is far from the original reference beam angle. The result is plotted in Fig. 10. For $\varphi=20^{\circ}, \Delta \theta_{o}$ is larger than $20^{\circ}$ for $\theta>110^{\circ}$, enough room to store the 1000 holograms in the previous example without hitting the side-peaks.

Another possible way to avoid cross-talk due to double grating formation, is to have at least one of the recording beams (preferably both) being only $e$ wave or $o$ wave during recording, and using only one polarization during reconstruction of the hologram. In a spatially multiplexed holographic memory, this means that we have to change the polarization as we scan the writing beams from one location to another. If the scanning is done by moving the crystal, we can accomplish this by using circularly polarized waves, and attaching a polarizer in front of the crystal.

It should be pointed out that as long as the writing beams are not eigenmodes ( $e$ and $o$ waves), double grating formation can occur, even when the crystal is not tilted 


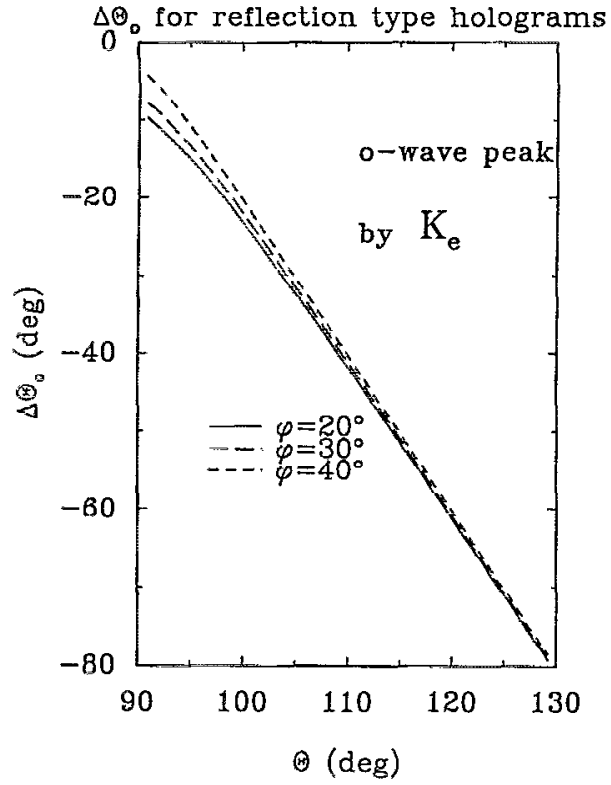

FIG. 10. Deviation angle of the 0 -wave side-peak from the recording reference beam angle as a function of $\theta$ for $\theta=20^{\circ}, 30^{\circ}$, and $40^{\circ}$.

with respect to the incident plane $(\varphi=0)$. In addition, if the signal beam is not a single plane wave but an image consisting of a spectrum of plane waves, then the condition $\varphi=0$ cannot be satisfied for most of the plane wave components. The results of this paper can be used to predict the expected cross-talk for this case as well, but the remedies that we outline above do not necessarily apply.

In some cases, the double grating effect might be of some use instead of a nuisance. As we have shown, by controlling the patterns that are $e$ wave and $o$ wave, it is possible to store two images (at the same reference beam angle) in one single exposure. We can select which image to be read-out by controlling the polarization at either the reference beam or reconstructed signal beam. An example of the usage of this double storage scheme is the representation of positive and negative numbers in optical neural net architectures (e.g., perception learning ${ }^{9,10}$ ) by the two orthogonal polarizations. This is shown in Fig. 11. Here we take the $e$-wave polarization to represent the "positive" part of the connection, and the $o$-wave polarization to represent the "negative" part. For each connection from the input plane to the output plane, there is a $\mathbf{K}_{e}$ and a $\mathbf{K}_{o}$ grating. Upon read-out, the signal from the input plane has both $e$-wave and $o$-wave components (this can be done by the same setup used in Fig. 7). The e-wave component, however, will diffract only off the $\mathbf{K}_{e}$ grating, while the $o$-wave component will diffract only off the $\mathbf{K}_{o}$ grating. The two components in the diffracted light are detected separately, and the difference of the two intensities are taken (electronically) to be the bipolar signal.

\section{ACKNOWLEDGMENTS}

The support of AFOSR and DARPA for this work is gratefully acknowledged. The authors thank Yong Qiao for his help and discussions.

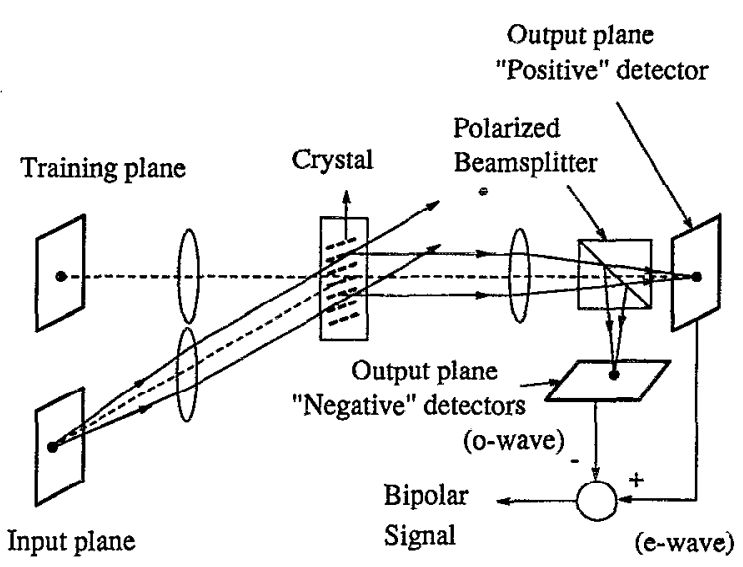

FIG. 11. Possible setup for implementing bipolar input/output signals in an optical neural network. Signals at both the training and input plane have $e$-wave and $o$-wave components.

\section{APPENDIX A: DERIVATION OF SLANT ANGLE}

Figure 12 shows the geometry used in our experiment. The signal beam $\mathbf{k}_{1}$ propagates along the $+x$ direction. Its $e$-wave polarization is in the $z$ direction, and its $o$-wave polarization is in the $y$ direction. For the reference beam, $\mathbf{k}_{2}$, the eigenmode polarizations are $\mathbf{q}(e$ wave) and $\mathbf{r}(0$ wave). It can be shown that $\mathbf{k}_{2}, \mathbf{q}$, and $\mathbf{r}$ are perpendicular to each other. Also, $\mathbf{r}$ lies in the $x-y$ plane. To see why this is true, consider rotating $\mathbf{k}_{2}$ about the $z$ axis until $\mathbf{k}_{2}$ lies in the $x-z$ plane. $\mathbf{q}$ and $\mathbf{r}$ will now rotate with $\mathbf{k}_{2}$. But when $\mathbf{k}_{2}$ lies in the $x-z$ plane, it is readily seen that $\mathbf{r}$ is parallel to the $y$ axis. This shows that $\mathbf{r}$ lies in the $x-y$ plane. Given this

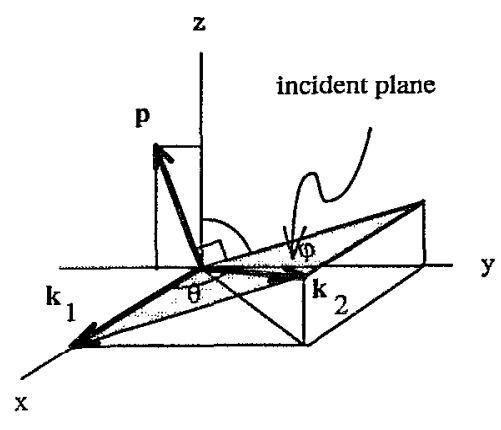

(a)

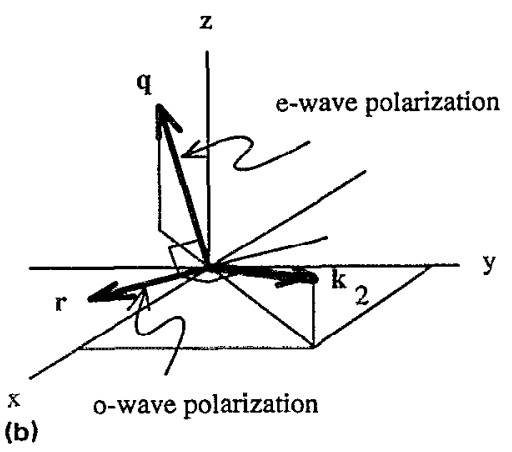

FIG. 12. (a) Geometry of reference $\left(\mathbf{k}_{2}\right)$ and signal $\left(\mathbf{k}_{1}\right)$ beams with respect to crystal axes. (b) Reference beam and its two eigenmode polarization directions, $\mathbf{q}$ ( $e$ wave) and $\mathbf{r}$ (o wave). 
fact, we see that $q$ lies in the plane formed by the $z$ axis and $k_{2}$. We are interested in the angle between $q$ and $p$, the direction perpendicular to the incident plane formed by $\mathbf{k}_{2}$ and $\mathbf{k}_{1}$.

Note that since $\mathbf{r}$ (thè $o$-wave polarization of $\mathbf{k}_{2}$, the reference beam) lies in the $x-y$ plane, and the $e$-wave polarization of $k_{1}$ (the signal beam) is in the $z$ direction, they are perpendicular to each other.

We have

$$
\mathbf{p}=(0,-\cos \varphi, \sin \varphi) .
$$

Thus the slant angle $\theta_{\text {slant }}$ is

$$
\theta_{\text {slant }}=\cos ^{-1}(\mathbf{u} \cdot \mathbf{p})=\cos ^{-1}\left(-u_{y} \cos \varphi+u_{z} \sin \varphi\right),
$$

where $\mathbf{u}$ is the unit vector parallel to q. It is easy to show that within a constant multiple

$$
\begin{aligned}
& q_{x}=-\sin \theta \cos \theta \cos \varphi \\
& q_{y}=-\sin ^{2} \theta \sin \varphi \cos \varphi \\
& q_{z}=\cos ^{2} \theta+\sin ^{2} \theta \sin ^{2} \varphi .
\end{aligned}
$$

This all we need to calculate the slant angle for various values of $\theta$ and $\varphi$.

\section{APPENDIX B: BRAGG MATCHING ANGLE}

The problem of finding the Bragg matching angle, given a grating vector, can be stated as follows: given a normal surface [in two-dimension (2-D)] described by the ellipse $\Gamma$

$$
\frac{x^{2}}{a^{2}}+\frac{y^{2}}{b^{2}}=1
$$

and a grating vector

$$
\mathbf{K}=\left(K_{x}, K_{y}\right)
$$

we want to find $(x, y)$ such that both $(x, y)$ and $\left(K_{x}+x, K_{y}+y\right)$ both lie on $\Gamma$.

We have

$$
\frac{x^{2}}{a^{2}}+\frac{y^{2}}{b^{2}}=1
$$

and

$$
\frac{\left(K_{x}+x\right)^{2}}{a^{2}}+\frac{\left(K_{y}+y\right)^{2}}{b^{2}}=1,
$$

which give us

$$
\left(\frac{2 K_{x}}{a^{2}}\right) x+\left(\frac{2 K_{y}}{b^{2}}\right) y+\left(\frac{K_{x}}{a}\right)^{2}+\left(\frac{K_{y}}{b}\right)^{2}=0 .
$$

Let

$$
u=\frac{x}{a}, \quad v=\frac{y}{b}
$$

and

$$
A=\frac{K_{x}}{a}, \quad B=\frac{K_{y}}{b},
$$

then

$$
u^{2}+v^{2}=1
$$

and

$$
2 A u+2 B v+A^{2}+B^{2}=0 .
$$

There are two solutions:

$$
\begin{aligned}
& x=\frac{K_{x}}{2} \mp \frac{K_{y}}{2}\left(\frac{a}{b}\right) \sqrt{\frac{4}{A^{2}+B^{2}}-1} \\
& y=\frac{K_{y}}{2} \pm \frac{K_{x}}{2}\left(\frac{b}{a}\right) \sqrt{\frac{4}{A^{2}+B^{2}}-1} .
\end{aligned}
$$

From these, we can calculate the angle between $(x, y)$ and the $x$ axis.

Given the recording geometry of the crystal, we can find $\mathbf{K}_{e}$ and $\mathbf{K}_{o}$ from $\theta$ and $\varphi$ [Eqs. (5) and (6)]. The problem then becomes finding the Bragg matching angle for $\mathbf{K}_{o}$ on the $e$-wave normal surface, and finding the Bragg matching angle for $\mathbf{K}_{e}$ on the $o$-wave normal surface (see Fig. 2). Once these are found, the corresponding incident angles using Snell's law can be found. We then subtract them from $\theta$ to obtain $\Delta \theta_{e}$ and $\Delta \theta_{o}$.

${ }^{1}$ N. V. Kukhtarev, V. B. Markov, S. G. Odulov, M. S. Soskin, and V. L. Vinetskii, Ferroelectronics 22, 949 (1979).

${ }^{2}$ F. H. Mok, M. C. Tackitt, and H. M. Stoll, Opt. Lett. 16, 605 (1991).

${ }^{3}$ H. Kogelnik, Bell Syst. Tech. J. 48, 2909 (1969).

${ }^{4}$ P. Gunter and E. Voit. Ferroelectrics 78, 51 (1988).

${ }^{5}$ S. I. Stepanov, M. P. Petrov, and A. A. Kamshilin, Sov. Tech. Phys. Lett. 3, 345 (1977)

${ }^{6}$ E. Voit, C. Zaldo, and P. Günter, Opt. Lett. 11, 309 (1986).

${ }^{7}$ F. Vachass and T. Y. Chang, J. Opt. Soc. Am. B 6, 1683 (1989).

${ }^{8}$ See for example, A. Yariv's Quantum Electronics, 3rd ed. (Wiley, New York, 1989).

${ }^{9}$ D. Psaltis, D. Brady, and K. Wagner, Appl. Opt. 27, 1752 (1988).

${ }^{10}$ J. H. Hong, S. Campbell, and P. Yeh, Appl. Opt. 29, 3019 (1990). 